Ann. Zootech., I972, 21 (2), 299-309.

NOTE

\title{
ESTIMATION DE LA COMPOSITION CORPORELLE D'AGNEAUX MERINOS D'ARLES ABATTUS A DES STADES DE CROISSANCE COMPRIS ENTRE LA NAISSANCE ET UN AN
}

\author{
M. PRUD'HON, Y. REYNE et X. GARAMBOIS \\ Station de Physiologie animale, \\ École nationale supérieure agronomique, \\ Centre de Recherches, I. N. R. A., \\ 34 - Montpellier
}

\section{RÉSUMÉ}

Quarante-six agneaux mâles et trente cinq femelles de race Mérinos d'Arles ont été sacrifiés et disséqués à $0,10,25,50,75$, 100, I 50,250 et 350 jours.

Une étude graphique, effectuée en coordonnées logarithmiques, a permis de mettre en évidence des relations linéaires étroites, quel que soit l'âge ou le poids des agneaux, entre le poids des trois grands tissus - osseux, musculaire, adipeux - et celui de certaines unités anatomiques, de nature homologue, facilement dissécables.

Cette note propose des équations permettant d'estimer, chez des agneaux Mérinos d'Arles sacrifiés entre la naissance et un an :

- le tissu squelettique à partir des poids du scapulum, du fémur ou de la somme des poids des canons ;

- le tissu musculaire à partir du poids des muscles sus-épineux, long vaste, grand psoas et du poids vif vide;

- les dépôts adipeux à partir du poids de la toilette et du poids vif vide. Dans ce cas l'estimation est beaucoup plus précise que l'estimation obtenue à partir du gras périrénal mais elle n'atteint pas la précision obtenue dans l'estimation du muscle et du squelette.

\section{INTRODUCTION}

L'étude de la composition corporelle des agneaux au cours de la croissance, qui renseigne sur les modalités de développement de l'organisme (BÉNÉVEnT, I97I), a aussi un intérêt du strict point de vue zootechnique : 
- d'une part elle permet de comparer, aux différents poids commerciaux d'abattage, les proportions respectives d'os, de muscles et de dépôts adipeux dont dépend assez étroitement la qualité des carcasses ;

- d'autre part, la comparaison d'animaux abattus à des stades successifs du développement permet d'estimer la composition tissulaire du croît, ce qui est utile dans les essais relatifs à la nutrition des agneaux (Vermores., Thivend et Thériez, I97o).

La seule méthode précise d'appréciation de la composition tissulaire de la carcasse est, actuellement, la dissection complète ; cette méthode longue et coûteuse est difficilement applicable, même à des fins purement expérimentales; de nombreux auteurs ont cherché à évaluer la composition corporelle des agneaux en tenant compte de leur poids ainsi que de la composition tissulaire d'une fraction de la carcasse : morceaux de découpe, unités anatomiques... (cf. revues de Flamant et Boccard, I966 et SeEbeck, I968). Étudiant des agneaux Ile-de-France, BocCARD et al., (I962) ont montré notamment qu'il existait une isométrie de développement entre la plupart des régions corporelles et l'ensemble de la carcasse. A poids égal, les proportions de muscles et d'os varient peu avec la vitesse de croissance (BocCard et DUPLAN, I96I; ElsLey, 1964). Il n'en est pas de même pour le tissu adipeux dont l'importance relative varie avec l'âge et le rythme de croissance et dont l'estimation demeure, même à un poids donné, extrêmement imprécise ainsi que l'ont souligné Flamant et Boccard (I966), Dumont (I97I).

Utilisant les données de dissection détaillée d'agneaux Mérinos d'Arles des deux sexes, abattus entre la naissance et l'âge d'un an, nous avons cherché une méthode d'estimation des principaux tissus - os, muscles squelettiques, dépôts adipeux — indépendante du stade d'abattage et de la vitesse de croissance des agneaux ; nous avons, en particulier, utilisé la propriété que possèdent certaines unités anatomiques ou unités de dissection d'avoir, par rapport à la masse totale des tissus de même nature, un coefficient d'allométrie qui demeure constant pendant une longue période de la croissance postnatale.

\section{MATÉRIEL ETT MÉTHODES}

Quarante-six agneaux mâles et trente cinq femelles de race Mérinos d'A $A$ les élevés en bergerie ont été sacrifiés et disséqués à l'âge de 0 , Io $\pm \mathrm{I}, 25 \pm \mathbf{I}, 50 \pm 2,75 \pm 2$, $100 \pm 2,150 \pm 2$, $25^{\circ} \pm \mathrm{I}$ et $35^{\circ} \pm \mathrm{r}$ jours.

TABLEAU I

Effectif et poids vif vide moyen des agneaux disséqués entre la naissance et un an

\begin{tabular}{|c|c|c|c|c|c|c|c|c|c|c|}
\hline \multicolumn{2}{|r|}{ Stades (jours) } & 0 & 10 & 25 & 50 & 75 & 100 & 150 & 250 & 350 \\
\hline \multirow{3}{*}{0} & Effectif...... & 2 & 4 & 7 & 7 & 6 & 10 & 6 & 2 & 2 \\
\hline & \multirow{2}{*}{ Poids vif vide \{} & 3918 & 6142 & 8746 & 13178 & 17790 & 19861 & 23355 & 35720 & 34153 \\
\hline & & 8,5 & 173 & 638 & 1846 & 1353 & 2498 & 2829 & 1203 & 1821 \\
\hline \multirow{3}{*}{ 웅 } & Effectif. . & 2 & 4 & 4 & 5 & 5 & 6 & 5 & 2 & 2 \\
\hline & \multirow{2}{*}{ Poids vif vide } & 3935 & 5824 & 8929 & 11867 & 15506 & 17908 & 22655 & 26746 & 31032 \\
\hline & & 121 & 223 & 207 & 627 & 1151 & 2784 & 1056 & 1685 & 1326 \\
\hline
\end{tabular}


Ces agneaux, choisis à la naissance, étaient élevés au sein du troupeau. Outre le lait maternel, ils avaient à leur disposition, dans un parc attenant, du foin de luzerne de bonne qualité et un aliment concentré à base d'orge. Le sevrage était pratiqué aux environs de 90 jours. Les pesées étaient hebdomadaires.

Les effectifs ainsi que les poids vifs vides moyens figurent au tableau $\mathbf{I}$.

Trente-quatre agneaux mâles et vingt sept femelles ont été disséqués en suivant aussi exactement que possible la technique décrite par BÉnt́vent (I968, I97I):

Les dissections musculaire, osseuse, ainsi que celle des dépôts adipeux de couverture et intermusculaires ont été opérées sur une demi-carcasse, des mesures préalables ayant montré que les régions symétriques ne différaient pas significativement de poids.

Les autres dépôts adipeux, les os canons et le squelette axial étaient intégralement prélevés.

Le reste de l'effectif a subi une dissection légèrement simplifiée et plus rapide :

- la dissection osseuse a été limitée aux 4 os canons et aux os longs d'une demi-carcasse ;

- les muscles de la tête et les muscles intercostaux, sus-costaux, intertransversaires des lombes, transverses du thorax et interépineux n'ont pas été isolés, ce qui représente une sousévaluation de 4 à 8 p. Ioo des muscles totaux; la sous-évaluation est moindre si l'on ne s'intéresse qu'aux muscles de la carcasse, tête exclue. Pour simplifier la terminologie utilisée nous appellerons "muscles totaux " le double du poids total des muscles disséqués.

Les différents dépôts adipeux ont été séparés en :

- gras de toilette (dépôt adipeux du petit epiploon),

- gras de ratis (dépôt adipeux du grand epiploon).

- gras de rognon ou périrénal,

- gras de la cavité pelvienne,

- gras de couverture ou sous-cutané,

- gras intermusculaire.

Les poids des contenus de l'estomac, de l'intestin et de la vessie ont été mesurés ; le poids vif vide a été estimé par différence ; le poids de carcasse a été obtenu par pesée à la sortie de la chambre froide, après vingt-quatre heures de ressuyage.

\section{Estimation des différents tissus}

Nous avons recherché des unités anatomiques ou des unités de dissection :

- bien définies sur le plan anatomique,

- faciles et rapides à isoler,

- ayant un coefficient d'allométrie constant par rapport au tissu dont on cherche à estimer le poids, de manière à obtenir une équation linéaire de type

$$
\log y=a+b \log x
$$

$y$ étant le poids du tissu à estimer (os totaux, muscle totaux, somme des dépôts adipeux), $x$ étant le poids de l'estimateur,

$b$ le coefficient d'allométrie du poids du tissu à estimer par rapport au poids du tissu estimateur.

Dans une étude graphique préliminaire, nous avions constaté que la plupart des os longs avaient, de la naissance à un an, un coefficient $b$ constant, la valeur de $b$ variant selon les os considérés; toutefois les nuages de points les moins dispersés correspondaient aux relations entre le squelette total d'une part et l'humérus, le radius cubitus, le scapulum, le fémur, la somme des 4 métacarpiens d'autre part ; par contre, la relation avec la première côte ou la dixième côte était plus lâche.

De la même façon le coefficient $b$ est relativement constant pendant cette même période de croissance lorsque l'on étudie les muscles groupés par région pour des animaux de même sexe. Toutefois, la dissection de l'ensemble d'une région peut être relativement longue et nous avons préféré prendre pour estimateurs les muscles sus-épineux, grand psoas, et long vaste qu'il est possible d'isoler rapidement et dont le poids, graphiquement et en échelle logarithmique, est en liaison linéaire étroite avec le poids total des muscles disséqués (cf. fig. I).

Parmi les 88 muscles disséqués, 4 autres muscles ont été testés graphiquement, en raison de leur emplacement et de leur facilité de prélèvement : extenseur antérieur du métacarpe, anconé externe, masse commune de la jambe, brachiocéphalique ; ils n'ont pas été retenus par suite d'une plus grande dispersion des points représentatifs.

Nous avons encore retenu le poids vif vide comme estimateur du poids des muscles squelettiques car il est souvent utilisé dans d'autres études (TULLOH, I963) et semble donner des résultats satisfaisants. 

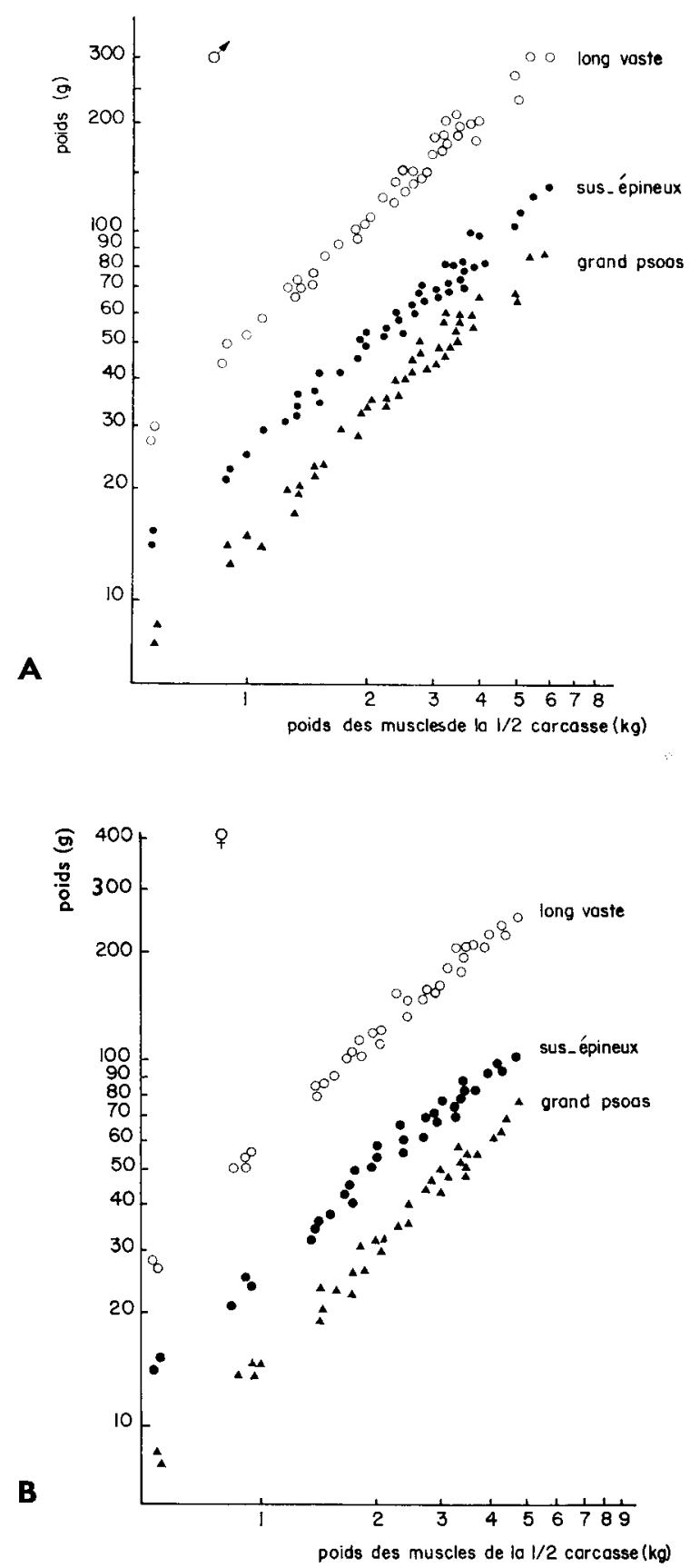

FIg. I ( $A$ et $B)$. - Relations entre le poids des muscles psoas, sus-épineux et long vaste et le poids des muscles d'une demi-carcasse pour des agneaux Mérinos d'Arles des deux sexes abattus entre la naissance et un an 
En ce qui concerne Ies dépôts adipeux, seul le gras de toilette semble, dans les deux sexes, évoluer de façon sensiblement linéaire par rapport à la somme des dépôts adipeux (cf. fig. 2).

Il est facile à isoler et son prélèvement ne déprécie pas la carcasse. Nous avons retenu également le poids vif vide et le gras de rognon, ce dernier étant fréquemment utilisé (cf. revue de Flamant et Boccard, r966 et Dumont, r97r).

Pour chaque tissu et chacun des sexes, nous avons établi, par la méthode des moindres carrés, des équations de régression simple entre un tissu et son estimateur, et de régression multiple entre un tissu et plusieurs estimateurs.

La comparaison des variances résiduelles a permis de juger de la précision des ajustements.

La comparaison des valeurs des coefficients d'allométrie $b$, obtenues pour les deux sexes a été effectuée en utilisant le test $t$.

$A$ posteriori nous avons calculé l'erreur relative moyenne

$$
\left(\frac{\mathrm{I}}{n} \sum_{1}^{n} \frac{\mid \mathrm{P}_{\text {calculé }} \text { - Pmesuré } \mid}{\text { Pmesuré }} \times \text { Ioo }\right)
$$

commise en utilisant les équations de prédictions pour des lots de Io agneaux ayant eu respectivement une croissance rapide, une croissance moyenne et une croissance lente. (Soit au cours de la période o-roo jours, une moyenne de 250 , 200 et $\mathrm{I} 30 \mathrm{~g}$ par jour pour les mâles; de 220 ,

A

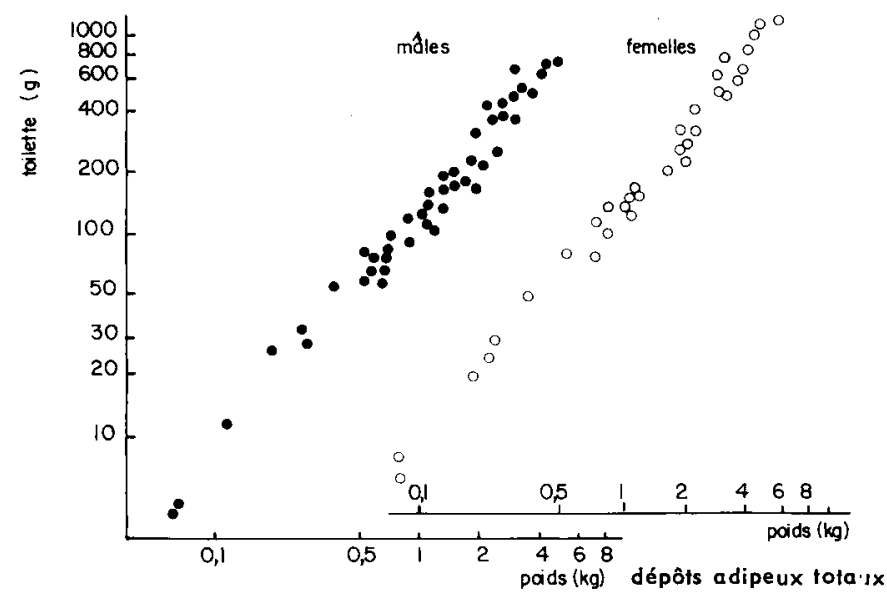

B

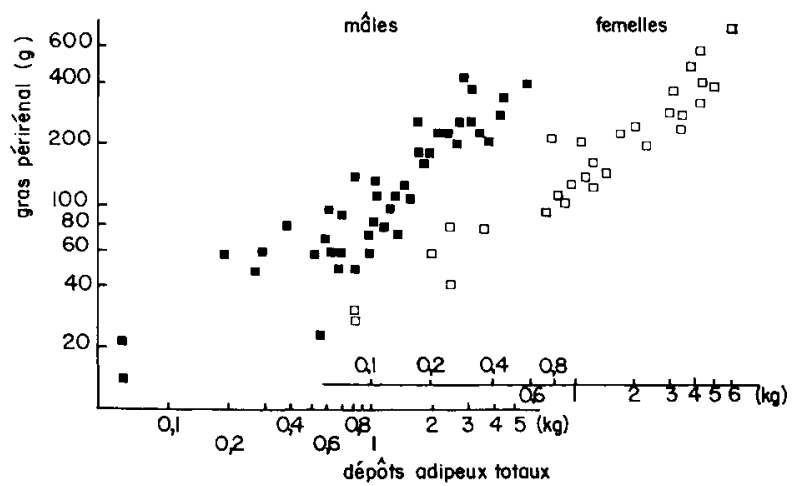

FIG. $2(A$ et $B)$. - Relations entre le poids de la "toilette "et du gras périrénal et la somme des dépôts adipeux dissécables d'agneaux Mérinos d'Arles des deux sexes abattus entre la naissance et un an 
I 80 et i zo g par jour pour les femelles). La mesure d'erreurs a porté, pour le muscle, sur les valeurs estimées par régression simple à partir du long vaste, régression multiple à partir du poids vif vide et du sus-épineux et régression multiple à partir du long vaste, du grand psoas et du sus-épineux.

En ce qui concerne le tissu adipeux, on a comparé les erreurs commises par estimation obtenue en utilisant une régression simple à partir du gras de toilette et en utilisant une régression multiple à partir du gras de toilette ct du poids vif vide Fig 2.

\section{RÉSULTATS ET DISCUSSION}

Dans le tableau 2 figurent les estimations des tissus squelettiques, musculaires et adipeux faisant intervenir des régressions simples. Les tableaux 3 et 4 donnent les équations de prédiction obtenues respectivement pour le tissu musculaire et le tissu adipeux à l'aide de régression simples et de régressions multiples.

\section{Squelette}

La comparaison des variances résiduelles ou des coefficients de détermination pour chacune des équations énumérées au tableau 2 nous montre que la somme des 4 canons n'est pas forcément l'estimateur le plus précis ; scapulum et fémur notamment sont plus valables. De plus, ainsi qu'on pouvait le prévoir compte tenu de la grande précocité de développement des os métacarpiens, la valeur du coefficient $b$ est assez éloignée de I pour ces os. Le scapulum ou le fémur sont à envisager comme estimateurs du squelette dans le cas où la dissection de morceaux tels que l'épaule ou le gigot sont utilisés pour l'estimation de la composition de la carcasse.

Dans le cas où l'on ne veut pas déprécier la carcasse, la somme des 4 canons peut être utilisée : c'est d'ailleurs l'estimateur le plus employé (PALsson, I940 ; Boccard, Dumont et Peyron, I958 ; Whiteman, Walters et Munson, ig66).

\section{Muscles}

L'étude des variances résiduelles nous montre, dans le cas ò̀ l'on utilise une équation de régression simple, que l'on obtient des ajustements meilleurs avec le long vaste et le sus-épineux qu'avec le grand psoas. Les valeurs du coefficient $b$ sont très proches de $I$ ce qui indique que l'augmentation relative de masse de l'un de ces muscles est pratiquement isométrique de l'augmentation relative de masse de la musculature totale. Dans le cas où l'on utilise le sus-épineux, une seule équation peut être utilisée pour les mâles et les femelles.

Enfin, l'utilisation du poids vif vide permet d'obtenir une précision identique à celle qui est obtenue avec chacun des deux muscles mais la détermination du poids vif vide nécessite la pesée des contenus stomacaux, intestinaux et vésicaux qui n'est pas rapide.

En utilisant conjointement le poids vif vide et le poids du sus-épineux (tabl 3 ) on abaisse assez fortement la variance résiduelle par rapport à l'équation de régression simple où intervenait le poids du sus-épineux seul. Par contre, l'utilisation d'une équation faisant intervenir les trois muscles sus-épineux, grand psoas et long vaste n'améliore pas davantage la précision des estimations (tabl. 3).

Les erreurs relatives moyennes obtenues en utilisant les différentes équations de prédiction sont respectivement de $5 \mathrm{p}$. Ioo pour les animaux à croissance lente, 3 à $4 \mathrm{p}$. Ioo pour les animaux à croissance moyenne, 5 à $8 \mathrm{p}$. Ioo pour les animaux à croissance rapide. Elles sont relativement faibles mais elles restent supérieures aux erreurs commises lors d'une dissection totale qui n'excèdent guère 2 p. Ioo si elle est exécutée soigneusement. Comme il était prévisible, les erreurs sont 
minimales pour les individus ayantuneintensité de croissance moyenne, car c'est dans la zone du point moyen que l'estimation du coefficient de régression est la plus précise.

\section{TABLEAU 2}

Corrélations et valeurs des paramètres des équations de régression simple $\left(\log y=a+b \log x_{n}\right)$ permettant l'estimation du poids des principaux tissus $(y)$ à partir de leurs estimateurs vespectifs $\left(x_{n}\right)$

\begin{tabular}{|c|c|c|c|c|c|c|c|c|c|}
\hline Tissu estimé & Estimateu & & Sexe & $n$ & $r_{x y}$ & $b y / x$ & $s_{b}$ & $\begin{array}{c}a \\
\text { constante }\end{array}$ & $\begin{array}{c}s_{r} \\
\text { Écart-type }\end{array}$ \\
\hline \multirow{8}{*}{$\begin{array}{l}\text { Squelette } \\
\text { total } \\
(y)\end{array}$} & \multirow{2}{*}{\multicolumn{2}{|c|}{$\begin{array}{l}\text { Poids des } \\
4 \text { canons }\end{array} \quad \mathrm{X}_{1}$}} & M & 34 & 0,980 & 1,474 & 0,052 & 0,539 & 0,096 \\
\hline & & & $\mathrm{F}$ & 27 & 0,980 & 1,492 & 0,060 & 0,493 & 0,091 \\
\hline & \multirow{2}{*}{$\begin{array}{l}\text { Poids du } \\
\text { scapulum }\end{array}$} & \multirow{2}{*}{$X_{2}$} & M & 34 & 0,993 & 0,843 & 0,018 & 4,508 & 0,059 \\
\hline & & & $\mathrm{F}$ & 27 & 0,994 & 0,846 & 0,018 & 4,467 & 0,049 \\
\hline & \multirow{2}{*}{$\begin{array}{l}\text { Poids du } \\
\text { fémur }\end{array}$} & \multirow{2}{*}{$\mathrm{X}_{3}$} & $\mathrm{M}$ & 34 & 0,992 & 1,049 & 0,024 & 2,932 & 0,063 \\
\hline & & & $\mathrm{F}$ & 27 & 0,995 & 0,051 & 0,022 & 2,913 & 0,047 \\
\hline & \multirow{2}{*}{$\begin{array}{l}\text { Poids du } \\
\text { radius-cubitus }\end{array}$} & \multirow{2}{*}{$\mathrm{X}_{4}$} & M & 34 & 0,989 & 1,101 & 0,034 & 3,026 & 0,069 \\
\hline & & & $\mathrm{F}$ & 27 & 0,989 & 1,222 & 0,032 & 2,719 & 0,072 \\
\hline \multirow{8}{*}{$\begin{array}{c}\text { Somme des } \\
\text { muscles } \\
\text { d'une demi- } \\
\text { carcasse } \\
(y)\end{array}$} & \multirow{2}{*}{$\begin{array}{l}\text { Poids du } \\
\text { Long vaste }\end{array}$} & \multirow{2}{*}{$X_{1}$} & M & 46 & 0,993 & 0,988 & 0,017 & 2,997 & 0,066 \\
\hline & & & F & 35 & 0,994 & 1,010 & 0,019 & 2,851 & 0,064 \\
\hline & \multirow{2}{*}{$\begin{array}{l}\text { Poids du } \\
\text { sus-épineux }\end{array}$} & \multirow{2}{*}{$X_{2}$} & $\mathrm{M}$ & 46 & 0,992 & 1,078 & 0,021 & 3,433 & 0,074 \\
\hline & & & $F$ & 35 & 0,994 & 1,079 & 0,020 & 3,439 & 0,064 \\
\hline & \multirow{2}{*}{$\begin{array}{l}\text { Poids du } \\
\text { grand psoas }\end{array}$} & \multirow{2}{*}{$x_{3}$} & $\mathrm{M}$ & 46 & 0,988 & 0,977 & 0,023 & 4,275 & 0,090 \\
\hline & & & $\mathbf{F}$ & 35 & 0,992 & 0,946 & 0,021 & 4,382 & 0,074 \\
\hline & \multirow{2}{*}{$\begin{array}{l}\text { Poids vif } \\
\text { vide }\end{array}$} & \multirow{2}{*}{$X_{4}$} & M & 46 & 0,993 & 1,006 & 0,018 & $-1,896$ & 0,068 \\
\hline & & & $\mathrm{F}$ & 35 & 0,994 & 1,015 & 0,019 & $-1,996$ & 0,062 \\
\hline \multirow{6}{*}{$\begin{array}{l}\text { Poids des } \\
\text { dépôts } \\
\text { adipeux } \\
\text { totaux } \\
(y)\end{array}$} & \multirow{2}{*}{$\begin{array}{l}\text { Poids de la } \\
\text { toilette }\end{array}$} & \multirow{2}{*}{$X_{1}$} & M & 46 & 0,987 & 0,851 & 0,021 & 2,819 & 0,163 \\
\hline & & & $\mathrm{F}$ & 35 & 0,990 & 0,823 & 0,021 & 2,892 & 0,163 \\
\hline & \multirow{2}{*}{$\begin{array}{c}\text { Poids de } \\
\text { gras périrénal }\end{array}$} & \multirow{2}{*}{$\mathrm{X}_{2}$} & M & 46 & 0,895 & 1,145 & 0,086 & 1,636 & 0,447 \\
\hline & & & F & 35 & 0,936 & 1,273 & 0,083 & 0,568 & 0,398 \\
\hline & \multirow{2}{*}{$\begin{array}{l}\text { Poids vif } \\
\text { vide }\end{array}$} & \multirow{2}{*}{$\mathrm{X}_{\mathbf{3}}$} & $\mathrm{M}$ & 46 & 0,948 & 1,656 & 0,084 & $-8,825$ & 0,320 \\
\hline & & & $\mathrm{F}$ & 35 & 0,971 & 1,901 & 0,082 & $-10,881$ & 0,270 \\
\hline
\end{tabular}


TABLEAU 3

Comparaison des différentes équations établies pour l'estimation du poids total de muscle de la demi-carcasse $(y)$

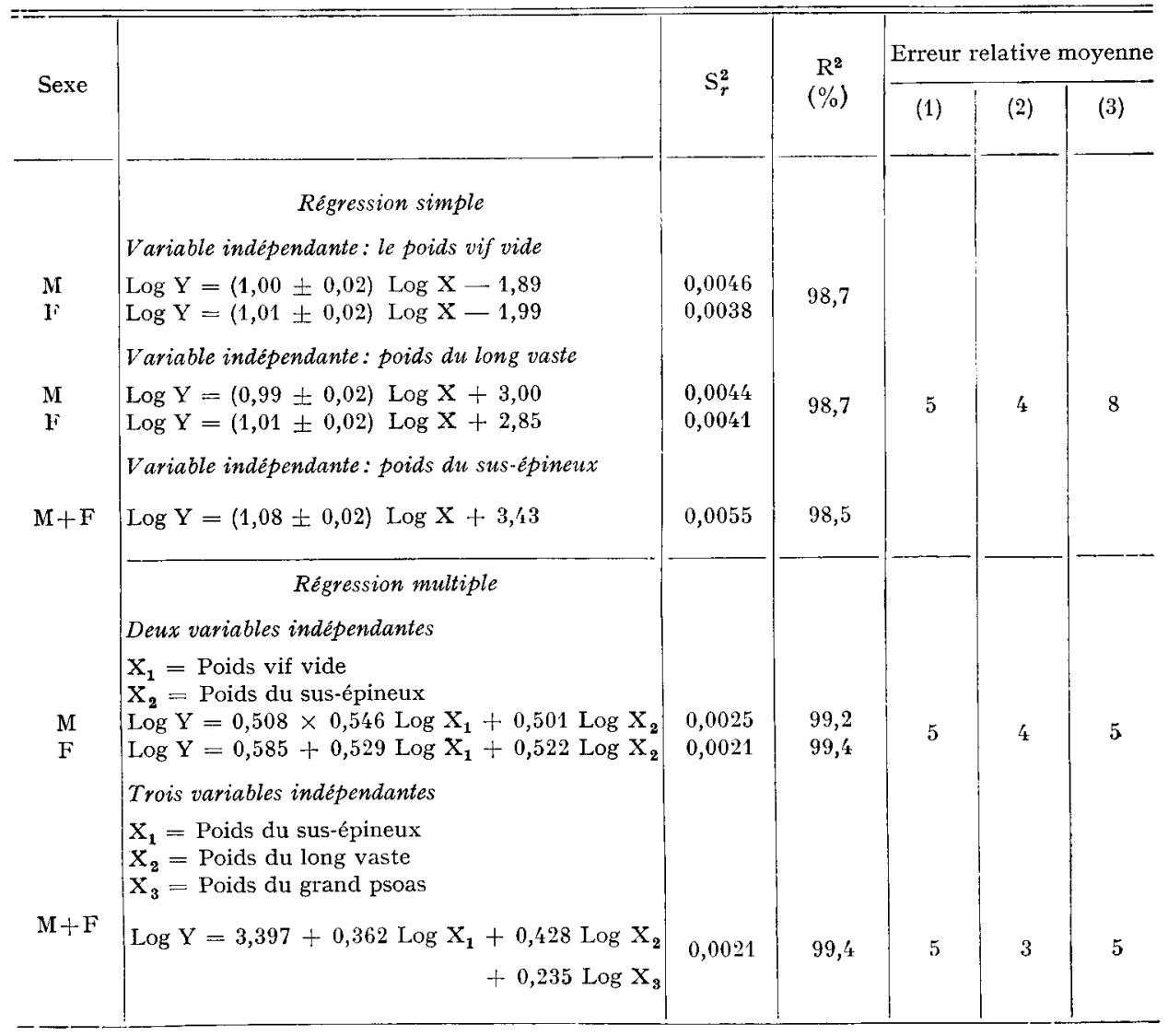

$\mathrm{S}_{r}^{2}=$ Variance résiduelle.

$\mathbf{R}^{2}=$ Coefficient de détermination.

(1) = Valeurs de l'erreur relative moyenne pour des agneaux à croissance lente.

(2) = Valeurs de l'erreur relative moyenne pour des agneaux à croissance moyenne.

(3) = Valeurs de l'erreur relative moyenne pour des agneaux à croissance rapide.

\section{Dépôts adipeux}

La toilette et le poids vif vide se révèlent de meilleurs estimateurs des dépôts adipeux totaux que ne l'est le gras de rognon ainsi qu'on peut le constater sur le tableau 2 ; le poids vif vide seul ne peut pas être considéré comme estimateur valable, l'écart-type résiduel étant trop important.

En combinaison avec la toilette dans une équation à deux variables explicatives (tabl, 4) le poids vif vide permet une légère diminution de la variance résiduelle, mais l'erreur relative ne descend guère en dessous de ro p. Ioo pour les lots qui n'excèdent pas une dizaine d'animaux.

Dans le cas où l'on s'intéresse exclusivement aux dépôts adipeux de la carcasse (gras péri- 
rénal, pelvien, intermusculaire et sous-cutané) la toilette demeure un bon estimateur de ces dépôts bien qu'elle ne fasse pas partie de ceux-ci; la relation toilette-dépôts adipeux de la carcasse est linéaire et les points représentatifs assez peu dispersés alors que la relation gras périrénal-dépôts adipeux de la carcasse présente un changement de pente et les points représentatifs une dispersion élevée.

\section{TABLEAU 4}

Comparaison des différentes équations établies

pour l'estimation du poids des dépôts adipeux totaux (dépôts périviscéraux inclus)

\begin{tabular}{|c|c|c|c|c|c|c|}
\hline \multirow{2}{*}{ Sexe } & & \multirow{2}{*}{$\mathbf{S}^{2}$} & \multirow{2}{*}{$\begin{array}{l}\mathrm{R}^{2} \\
(\%)\end{array}$} & \multicolumn{3}{|c|}{ Erreur relative moyenne } \\
\hline & & & & (1) & (2) & (3) \\
\hline & $\begin{array}{l}\text { Régression simple } \\
\text { Variable indépendante: poids de la toilette }\end{array}$ & & \multirow[b]{2}{*}{97,9} & \multirow{4}{*}{13} & \multirow{4}{*}{9} & \multirow{4}{*}{10} \\
\hline $\begin{array}{l}\mathrm{M} \\
\mathrm{F}\end{array}$ & $\begin{array}{l}\log \mathrm{Y}=(0,85 \pm 0,02) \quad \log \mathrm{X}+2,82 \\
\log \mathrm{Y}=(0,82 \pm 0,02) \log \mathrm{X}+2,89 \\
\text { Variable indépendante: poids du gras périrénal }\end{array}$ & $\begin{array}{l}0,0264 \\
0,0265\end{array}$ & & & & \\
\hline $\begin{array}{l}\mathrm{M} \\
\mathrm{F}\end{array}$ & $\begin{array}{l}\log Y=(1,14 \pm 0,09) \quad \log X+1,64 \\
\log Y=(1,27 \pm 0,08) \quad \log X+0,57\end{array}$ & $\begin{array}{l}0,1999 \\
0,1580\end{array}$ & 86,4 & & & \\
\hline \multirow[t]{2}{*}{$\begin{array}{l}\mathrm{M} \\
\mathrm{F}\end{array}$} & $\begin{array}{l}\text { Variable indépendante: poids vif vide } \\
\log \mathrm{Y}=(1,65 \pm 0,08) \log \mathrm{X}-8,82 \\
\log \mathrm{Y}=(1,90 \pm 0,08) \log \mathrm{X}-10,88\end{array}$ & $\begin{array}{l}0,1023 \\
0,0735\end{array}$ & 94,0 & & & \\
\hline & $\begin{array}{l}\text { Régression multiple } \\
\text { Deux variables } \\
\mathrm{x}_{1}=\text { Poids vif vide } \\
\mathrm{X}_{2}=\text { Poids de la " toilette" }\end{array}$ & & & & & \\
\hline $\begin{array}{l}\mathrm{M} \\
\mathrm{F}\end{array}$ & $\begin{array}{l}\log Y=0,945+0,265 \log X_{1}+0,713 \log X_{2} \\
\log Y=0,389 \log X_{1}+0,673 \log X_{2}-0,020\end{array}$ & $\begin{array}{l}0,0255 \\
0,0202\end{array}$ & $\begin{array}{l}97,9 \\
97,9\end{array}$ & 11 & 8,9 & 10 \\
\hline
\end{tabular}

$\mathrm{S}_{r}^{2}=$ Variance résiduelle.

$\mathbf{R}^{2}=$ Coefficient de détermination.

(1) = Valeurs de l'erreur relative pour les agneaux à croissance lente.

(2) = Valeurs de l'erreur relative pour les agneaux à croissance moyenne.

(3) = Valeurs de l'erreur relative pour les agneaux à croissance rapide.

Les équations permettant d'estimer le poids des dépôts adipeux de la carcasse $(y)$ à partir du poids de la toilette $(x)$ sont respectivement :

pour les mâles :

$$
\log y=(0,85 \pm 0,026) \log x+2,56\left(\text { avec } \mathrm{R}^{2}=97,3\right. \text { p. то0) }
$$

pour les femelles :

$$
\log y=(0,80 \pm 0,027) \log x+2,75\left(\text { avec } \mathrm{R}^{2}=96,4\right. \text { p. IOO) }
$$

L'estimation des dépôts adipeux de la carcasse peut donc être obtenue à partir du poids de la toilette avec une précision très sensiblement voisine de celle de l'ensemble des dépôts adipeux de l'Agneau. 


\section{CONCLUSIONS}

Nous avons recherché une méthode rapide et suffisamment précise d'estimation de la composition tissulaire d'agneaux, couvrant une grande échelle de poids et indépendante de la vitesse de croissance des individus; à cet effet nous avons retenu comme critère de choix des variables " estimatrices » la constance du coefficient d'allométrie.

Nous nous sommes attachés à donner une mesure de la précision de nos estimations en calculant, pour les équations les plus satisfaisantes, le coefficient de détermination; ceci permet la comparaison aux données de la littérature qui comportent ce paramètre.

Dans l'ensemble nous avons obtenu des ajustements aussi précis que ceux qui sont couramment utilisés en ce qui concerne le muscle et l'os et plus précis dans le cas des dépôts adipeux.

Nous avons montré, dans le cas des agneaux Mérinos d'Arles de notre élevage, que les meilleures estimations du poids du squelette et des muscles pouvaient être obtenues respectivement à partir du poids du scapulum et du sus-épineux; l'estimation des dépôts adipeux totaux et des dépôts adipeux de la carcasse, obtenue en utilisant le poids de la toilette, est relativement satisfaisante à tous les stades de la croissance. Elle est meilleure que l'estimation à partir du gras périrénal mais sa précision n'atteint pas malgré tout la précision obtenue pour le squelette et les muscles.

\section{Reçu pour publication en février 1972.}

\section{SUMMARY}

\section{ESTIMATION OF THE BODY COMPOSITION}

\section{OF ARLES MERINO LAMBS AT DIFFERENT GROWTH STAGES BETWEEN BIRTH} AND THE AGE OF ONE YEAR

Eighty one Avles Merino lambs, 49 males and 35 females, were slaughtered and dissected at the age of $0,10,25,50,100,150,250$ and $35^{\circ}$ days.

By means of graphs plotted in log-log coordinates it was possible to demonstrate close linear relationships, whatever the age or the weight of the animals, between the weight of the three main tissues (bone, muscle, fat) and that of some easily dissectable anatomical units of homologous nature.

The present note proposes equations allowing the following estimations in Arles Merino lambs slaughtered between birth and the age of one year:

- bone tissue, from the weights of the scapulum and the femur or from the total weights of the cannons,

- muscular tissue, from the weight of the muscles $M$. supraspinatus, M. Biceps femoris, $M$. psoas major and from the empty body weight,

- adipose tissue, from the weight of the caul and from the empty body weight. This estimation is much more exact than that obtained from the weight of the perinephric fat, but it does not reach the accuracy of the muscle and bone estimation.

\section{RÉFÉRENCES BIBLIOGRAPHIQUES}

BÉnÉvent M., I968. Guide pour la dissection et l'identification des principaux muscles de la carcasse chez le Mouton. Ann. Biol. anim. Bioch. Biophys., 8, I47-193.

BÉnÉvent M., I97x. Croissance relative, pondérale, postnatale, dans les deux sexes, des principaux tissus et organes de l'Agneau Mérinos d'Arles. Ann. Biol. anim. Bioch. Biophys., 11, 5-39. 
Boccard R., DUplan J.-M., I96r. Étude de la production de la viande chez les ovins. III. Note sur l'influence de la vitesse de croissance sur la composition corporelle des agneaux. Ann. Zootech., 9, $355-363$.

Boccard R., Dumont B.-L., Lefebvre J., I962. Étude de la production de la viande chez les ovins. V. Note sur la croissance relative des régions corporelles de l'Agneau. Ann. Zootech., 11, $257-262$.

Dumont B.-L., r97r. Qualités des ovins de boucherie. Pâtre nº 183, 4-12.

Elsley F. H. W., McDonald I., Fowler V. R., I964. The effect of plane of nutrition on the carcasses of pigs and lambs when variations in fat content are excluded. Anim. Prod., 6, I4I-I54.

Flamant J. C., Boccard R., I966. Estimation de la qualité de la carcasse des agneaux de boucherie. Ann. Zootech., 16, 4I-63.

Palsson H., I940. Meat qualities in the sheep with special references to scottish breeds and crosses. J. agric. Sci. Camb. 29, 560.

Sefbeck R. M., I968. Developmental studies of body composition. Anim. Breed. Abstr., 36, I67-I71. Tulloh N. M., 1963. Symposium carcass composition and appraisal of meat animals. Commonw. scient. ind. Res. Org. Australie.

Vermorel M., Thivend P., Theriez M., I970. Utilisation énergétique de l'orge, du blé, du maïs et du sorgho par l'Agneau en croissance. Ann. Zootech., 19, 46I-464.

Whiteman J. V., Walters L. E., Munson A. W., ig66. Some relationships between lamb growth and carcass composition and among various measures of carcass merit. Misc. Publs. Okla. agric. Exp. Stn., 78, 40-49, in Anim. Breed. Abstr. 35, n० $4 \mathrm{I} 3$. 